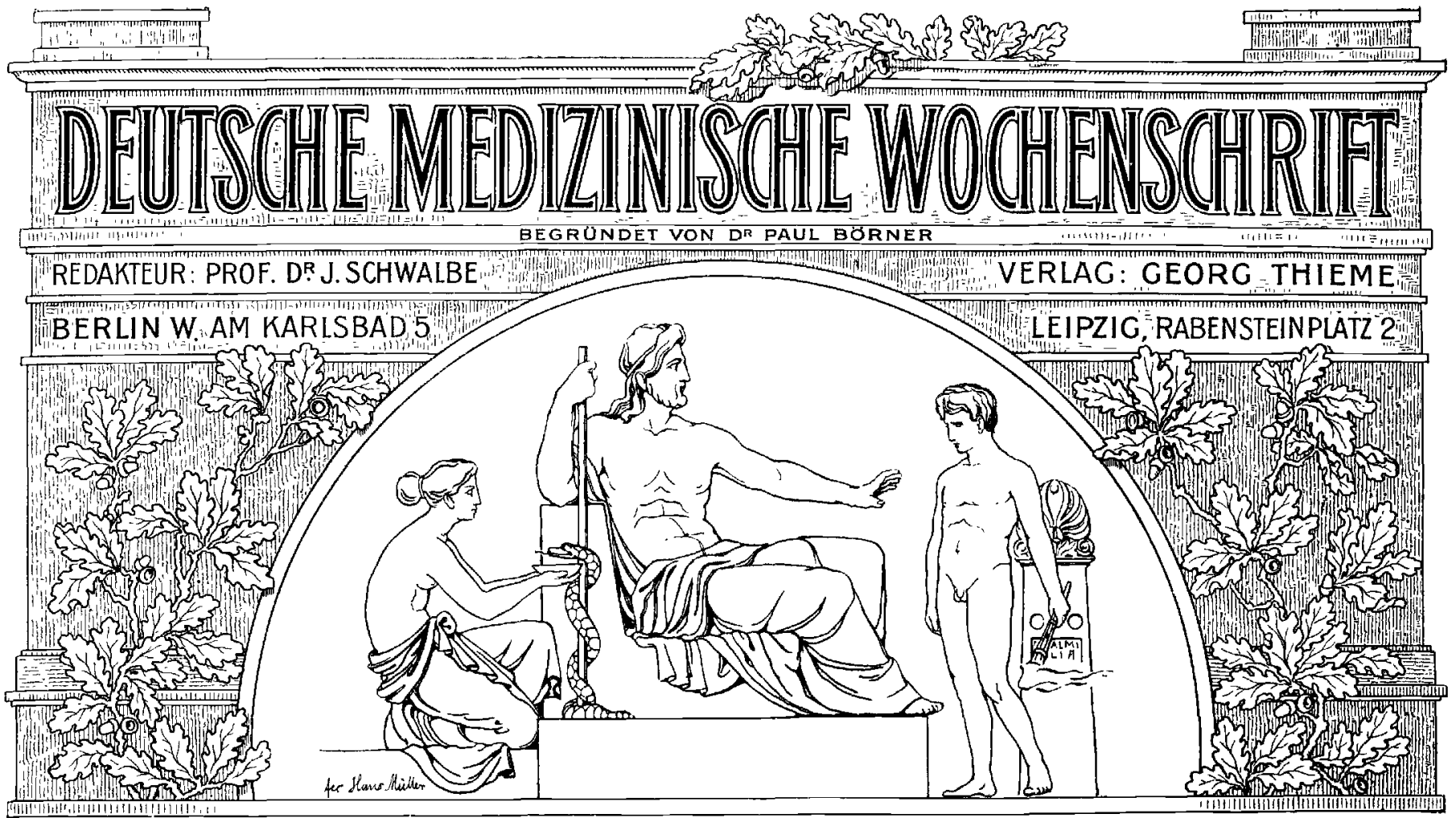

No. 47 .

Donnerstag, den 21. November 1907.

33. Jahrgang.

Aus der Universitäts-Klinik und -Poliklinik für psychische und Nervenkrankheiten in Göttingen.

\section{Die Behandlung der arteriosklerotischen Atrophie des Großhirns.}

Klinischer Vortrag.

Von A. Cramer.

M. H.! Wir haben uns in den letzten Stunden mit den verschiedenartigen Zustandsbildern beschäftigt, welche auf dem Boden der arteriosklerotischen Atrophie des Großhirns vorkommen können. Ich darf wohl kurz das Wesentliche, was wir an der Hand unserer Beobachtungen festgestellt haben, rekapitulieren: Wir haben gesehen, dab die Patienten sich meist am Ende des vierten oder im fünften Lebensjahrzehnt befanden, daß aber auch im sechsten Lebensjahrzehnt die Krankheit keine Seltenheit ist und daß sie auch noch später, wenn auch meist weniger charakteristisch zur Entwicklung kommen kann.

Die Prodrome, welche häufig nicht genügend beachtet oder auch übersehen werden, bestehen in der Trias: Kop f schmer$z e n$, Schwindel und zunehmende Gedächtnisschwäche. Diese Erscheinungen, zeitweise mehr, zeitweise weniger hervortretend, zeigen aber im allgemeinen die Tendenz, an Intensität und Hartnäckigkeit zuzunehmen. Alsdann macht sich eine Ver änderung der psychischen Individualität geltend, die je nach der persönlichen Eigenart des einzelnen Falles in depressiven oder exaltativen, vorübergehenden oder dauernden Stimmungsschwankungen oder in einer zunehmenden Stumpfheit zum Ausdruck kommt und je nach der Art der Ausbreitung und Lokalisation der Arteriosklerose im Gehirn mit monoplegischen, hemiplegischen oder aphasischen Erscheinungen verbunden sein oder die Symptome der Spätepilepsie aufweisen oder auch leichte oder schwere BewuBtseinsstö. r ungen darbieten kann und nicht selten von Zuständen eigentümlicher Desorientiertheit begleitet ist. Auch der amnestiche Symptomenk omplex kann sich gelegentlich einstellen.

Alle diese Erscheinungen können einen mehr stationären oder mehr flüchtigen Charakter zeigen. Im letzteren Falle kehren sie aber häufig ebenso plötzlich, wie sie verschwunden sind, wieder. Auch in den Fällen, wo ausgesprochene hemiund monoplegische Erscheinungen fehlen, findet sich immer eine Reihe von Symptomen, welche so ausgesprochen fast nul bei organischen Erkrankungen des Gehirns vorkommen: Fazialisparesen, träge reagierende Pupillen, leichtere oder schwerere artikulatorische Sprachstörung, verwaschene, sehr verlangsamte Sprache, Zeichen von amnestischer Aphasie, flüchtige Heminaopsien, Ophthalmoplegien, Steigerung der Reflexe, Ungleichheiten der Reflex, angedeutete Tastlähmungen, gelegentlich auch spastische Symptome und Gangstörungen. Also im allgemeinen Ausfallserscheinungen, welche auf eine organische Erkrankung einzelner Neurone hinweisen.

Dazu kommen bei der äußeren Untersuchung der Arterien nicht selten, aber durchaus nicht immer die fühlbaren $\mathrm{Z} \theta \mathrm{ich}$ en der Arteriosklerose an den Radiales, Temporales und Brachiales zum Ausdruck. Das Herz weist nicht selten eine Vergrößerung nach links auf, der Puls ist gespannt, im Urin findet sich häufig Eiweif. Auch wird gelegentlich Diabetes beobachtet, hier in Göttingen allerdings seltener als an den Orten, wo ich früher wirkte. Häufig machen die Patienten mit Beginn ihrer Erkrankung äußerlich einen sehr gesunden, robusten, widerstandsfähigen Eindruck, oft sind sie sehr gut genährt, gelegentlich findet man auch eine vergrößerte Leber.

Unter den Ständen und Berufsarten, die am meisten betroffen werden, stehen Gastwirte, Schauspieler, Offiziere, Bankiers, Parlamentarier, Mitglieder von Aufsichtsräten obenan. Es scheinen aber auch solche Individuen leicht befallen zu werden, welche häufig unter dem Einfluß von starken Affekten, von Kummer und Sorgen gestanden haben, auch übertriebener Sport scheint ätiologisch in Betracht zu kommen. Viele der Patienten sind, wenigstens früher, sogenannte Kraftnaturen gewesen; trotzdem wäre es aber falsch, die Krankheit allein auf Trinkexzesse und Tafelfreuden zurückzuführen, wie das gelegentlich geschieht, wenn ich auch nicht leugnen kann, daß sich unter meinen Patienten viele sogenannte starke Essel' finden.

Bei Besprechung der Therapie dieser, wie mir scheint, in ihrer Verbreitung noch zunehmenden Krankheit muß ich zunächst die Prophylaxe einer Erörterung unterziehen. Ich habe schon wiederholt darauf hingewiesen, wie schwierig es ist, über die Ursachen der Arteriosklerose ins klare zu kommen. Wenn wir alle die Schädlichkeiten, welche auch nach Angabe ernster Autoren Arteriosklerose hervorrufen, vermeiden 
wollten, würden wir uns überhaupt nicht mehr regen liönnen und vielleicht trotzdem dieser Krankheit verfallen sein.

Das, was wir vermeiden können, ist die Syphilis, der übermäßige Genuß von Alkohol, die zu starke Huldigung der Tafelfreuden und die übertriebene sportliche Betätigung. Die Personen, welche aus Familien stammen, in denen eine ausgesprochene Neigung zu Arteriosklerose vorhanden ist, werden ganz besonders vorsichtig in dieser Hinsicht sein müssen. Auch ist es für den Hausarzt eine ernste Pflicht, in den Fällen, wo sich auch nur die leisesten prämonitolischen Symptome zeigen, wit aller Energie auf eine sofortige Aenderung der Lebensweise zu drängen. Je eher mit der Therapie eingegriffen wird, um so mehr verspricht, wie bei jeder anderen Krankheit, auch hier die Behandlung Erfolg. Zeigt sich also bei einem Patienten die ominöse Trias: Kopfschmerz, Schwindel und beginnende Gedächtnisschwäche, lassen irgendwelche anderen Erkrankungen eine Erklärung für diese Symptome nicht zu, dann müssen wir mit unserer Behandlung beginnen. Die erste Bedingung ist, dab wir das Organ, welches erkrankt ist, das Gehirn, wie jedes andere erkrankte Organ, ruhig stellen. Dies können wir nur erreichen durch Entfernung des Patienten aus seinem Berufe mit allen seinen Aufregungen und oft auch aus der Familie, welche häufig nicht minder erregende Momente in Menge für den Kranken birgt.

Wenn uns das gelingt, ist schon viel gewonnen. Ich mache Sie aber darauf aufmerksam, daß diese „Reizentfernung“" oft aus äuferen Gründen auf die größten Schwierigkeiten stößt. Die Patienten sitzen in einer Menge von wichtigen Aemtern und Geschäften, es stehen Verluste an Erwerb, Ansehen und Stellung in Gefahr, die Kranken und ihre Familie glauben nicht an den Ernst der Situation, und so scheitert unser Bestreben, wenn uns nicht ein autoritativer Ausspruch eines angesehenen Arztes hilft. Aber auch dann haben wir noch nicht gewonnen. Wenn nach einigen Wochen der Aussparınung eine Besserung sich bemerklich macht, stürzt sich unser Patient sofort wieder in seine Geschäfte, und jetzt erst, wenn ein völliger Zusammenbruch, ein ausgesprochener Insult als alarmierendes Zeichen die Patienten und den Angehörigen erschreckt, wird dem Arzte besser gefolgt. Doch auch jetzt noch können wir Ueberraschungen erleben, habe ich doch oft gesehen, dab selbst nach solchen Attacken die Patienten, kaum etwas erholt, sich in neue geschäftliche Unternehmungen stürzten, sich ins Parlament wählen ließen oder eine neue Ehe eingingen, um nach kurzer Zeit todkrank und unheilbar ihrem Schicksal zu verfallen.

Die Verhältnisse können aber auch so liegen, daß außelordentlich widrige Lebensverhältnisse dem Kranken trotz aller unserer Bemühungen immer aufs neue ein reiches Maß von Kuinmer und Sorge schaffen, z. B. mißratene Kindel, ungünstige geschäftliche Konjunktur, drohender Bankerott, drohender Verlust der Stellung (Schauspieler, Offiziere). Trifft in diesen Fällen noch die Unvernunft der Angehörigen, welche den Ernährer bis auf den letzten Tropfen ausnutzen wollen, zusammen, so sind meistens unsere therapentischen Versuche vergeblich, die beginnende Krankheit kommt nicht zum Stillstand, sondern entwickelt sich rapid weiter.

Gelingt es uns abel, eine Ruhigstellung des Gehirns zu erzielen, so haben wir die Aussicht, eine weitgehende Besserung zu erreichen, ja die Krankheit zum Stillstand zu bringen und fast eine Restitutio ad integrum herbeizuführen, wenn wir die Patienten möglichst früh in Behandlung bekommen.

Wie wir bestrebt sind, durch Entfernung von den häuslichen, amtlichen und geschäftlichen Reizen die starke Inanspruchnahme des Gefäßsystems (durch die damit verbundenen Affekte) zu beseitigen, so müssen auch unsere weiteren therapeutischen Maßnahmen darauf Bedacht nehmen, nach Möglichkeit eine schädliche Ueberanstrengung des Zirkula. tionsapparates zu verhindern. Dazu gehört in erster Linie das Verbot des Genusses alkoholischer Getränke. Wenn ich es auch durchaus nicht für wissenschaftlich bewiesen halte, daß der Gellub geistiger Getränke, der sich in mäßigen, individuell abzumessenden Glenzen hält, irgend etwas mit der Arteriosklerose zu tun hat, so kommt doch bei unseren Pa- tienten in Betracht, dak gar mancher sich darunter befindet, bei dem das, was or für mälig hält, bereits viel zu viel ist, und dab in der Regel, wenn auch nur eine leichte Besserung sich geltend macht, die erste beste Gelegenheit benutzt wird, um über die Stränge zu schlagen. Man hat den Patienten nul sicher in der Hand, wenn nlan Alkohol in jeglicher Form verbietet. Für einen Ersatz muß man sorgen. Hierfül sind besonders die alkoholfreien Getränke und die Mineraltafelwässel geeignet. Ich empfehle hier gewöhnlich den Apfeltrank von Grotefend in Göttingen, voll dem ich durchl die Liebenswürdigkeit von Geheinnat Wallach eine chemische Analyse besitze, und je nach Lage des Falles, kgl. Selters, Kaiser-FriedrichQuelle oder eins der bekannteren Gichtwässer, wenn entsprechende Komplikationen bestehen. Aber auch Fruchtsäfte und frisch bereitete Zitronenlimonade wird zweckmäßig zur Abwechslung gereicht.

Mindestens ebenso wichtig wie die Uebel'wachung der Getränke, welche der Patient zu sich nimmt, ist die Regelung der Ernährung und strenge Ueberwachung der Diät. Denn gerade hier welden namentlich bei den Vielessern dem Gefäßsystem oft ungeheuere Strapazen zugemutet. Man sieht nicht selten die Patienten schwer atmend und keuchend, ja transpirierend, vom Tische aufstehen. Die Blutdruckmessung ergibt eine viel höhere Steigerung, als sie sich bereits normaliter nach den Mahlzeiten befindot. Schreibt man nicht ganz genau Quantum und Art der Diat vor, so hat man immel. wieder mit starken Ueberladungen des Magens zu rechnen. Ueber die Art dieser Diät habe ich schon oft mit Ihnen ge. sprochen, ich will destalb nur heute wieder betonen, daß wil alle Speisen vermeiden, die stark blähen und einen unnötigen Ballast für den Magen-Darmtractus darstellen, und stets darauf achten müssen, daß nicht zuviel auf einmal gegessen wird.

Aehnlich genau müssen wir auch auf die Bewegungsleistungell, welche der Patient unternimmt, achten. Wil. wissen ja, daß jede Alt ron Bewegung den Blutdruck steigert und das Gefäbsystem mehr oder weniger in Anspruch nimmt. Wenn es nun auch nicht notwendig ist, da\} wir in den frischen, leichteren Fällen den Patienten zur Bettruhe verdammen, so müssen wir doch genau zn erforschen suchen, was er sich in diesel Beziehung alles zumutet. Wir werden dann sehen, dak der eine "Müllert", del andero morgells ,seine Klimmzüge" macht oder "angestrengt rudert, oder reitet und dgl. mehr; auch erfahren wir, daß die Patienten sich den anstrengendsten und eingreifendsten hydrotherapeutischen Prozeduren unterziehen, um den "nervösen Zustand" zu bekämpfen. Es ist selbstverständlich, daß wir alledem durch ein kategor.isches Veto ein Ende boreiten. Erw inscht ist dagegen ein möglichst ausgedehntel Aulenthalt in frischer Luft, del in leichtelen Fällen mit genau dosierten Spaziergängen zu ebener Erde verbunden werdell kann. Warnen nı üsen wir die Patienten vol jeder folclerten Körpelbewegung, z. B. laschem Steigen von Treppen, raschem Laufen auf der Bahn, lautem allhaltenden Sprechen, Kohabitationen und dgl. Auch empfiehlt es sich, wenn wir die Patienten in ein Bad schicken, sie darauf aufmerksam zu machen, daß sie sich kühles Wetter zur Reise aussuchen, weil das lange Sitzenl im überfüllten Abteil bei starker Hitze und Schwule außerordentlich große Anforderungen an Herz und Gefäßsystem stellt.

Besonders wichtig ist in allen Fällen die Ueberwaclıung der Darmtätigkeit. Es ist Ihnen ja bekannt, daß das starke Pressen zur Stullentleerung schon manchem Patienten verhängnisvoll geworden ist In den Fällen, wo eine chronische Stuhlverstopfung besteht, tut nıan gut, zun̈̈chst Oeleinläufe nach Ebstoin zu versuchen; allerdings muß man sich hüten, die Kul zu lange fortzusetzen, weil man sonst die Patienten zu stark helunterbringt. Länger wie 14 Tage, höchstens drei Wochen, habe ich die Kul nie durchgeführt. In den meisten Fällen, und namentlich da, wo eine gewisse Plethora, verbunden mit Fettleibigkeit, vorhanden ist und Vergröherung der Leber besteht, ist eine fiur mit Karlsbader Mählbrunner fast immer von ausgezeichnetem Erfolg. Ich gehe gewóhnlich so vor, dal ich den Brunnen inorgens nüchtern in der Menge von ein bis zwei. ill seiternen Fallen anch bis zu drei drittel Liter etwa vier Wochen lang nehmen lasse, alsdann nache ich einen 
Monat Pause, um die Knr von neuem zu beginnen. In der Zwischenzeit lasse ich, wenn sich die Stuhlentleerung nicht glatt von selbst reguliert und wenn reichliche Obstdarreichung am Abend versagt, milde Abfühımittel geben: Rheum, Tamarindenkonserven, Regulin, Cascara sagrada und andere in stetem Wechsel. In einzelnen Fällen habe ich ein halbes Jahr lang und darüber mit sehr gutem Erfolge jeden zweiten Monat Karlsbader Wasser trinken lassen. Oft schwinden schon nach den ersten acht Tagen die Zistände von Benommenheit und leichter Desorientiertheit. Sehr zu empfehlen ist, wonn die äußeren Verhältnisse es erlauben und der Fall nicht zll weit fortgeschritten ist, eine $K l \mathrm{r}$ in Karlsbad selbst. Selbstverständlich ist, daß diese Kuren ärztlich üborwacht werden müssen.

Handelt es sich um Fälle, wo bereits ein leichter Insnlt dagewesen ist oder gar bereits monoplegische oder hemiplegische oder aphasische Störungen oder andere El'scheinungen eines Hirnherdes sich geltend machen, so ist es weselltlich, daß wir für längere Zeit für inöglicliste Ruhe, und llamentlich für Vermeidung stärlkerer Blutdruckschwankungen sorgen. Wir legen also den Kranken zu Bett und lassen ihn solange liegen, bis wir eine gewisse Konsolidierung des akuten zerebralen Prozesses, der zur Blutung gefuihrt hat, erwarten können. Alsdann fangen wir schrittweise ulld vorsichtig an, den Kranken wieder an Bewegung zu gewöhnen.

In jedem Falle ist besonders wichtig, sei er leichter oder schwer, sei er frischer oder älter, daß wir sofort mit der me dikamentösen Therapie beginnen. Diese besteht in der Darreichung von Jodpräparaten. In den allermeisten Fällen kommi man mit Jodkalium oder Jodnatrium, das in einfacher Lösıng gegeben wird, ans. lch fange mit $1 / 4 \mathrm{~g}$ an und steige, wenn es gut vertragen wird, rasch anf zwei, auch drei, ja sogar vieı Gramm pro die. lnı allgemeinen kommt es darauf an, die Joddarreichung niclit zu sehr zll forcieren. Denn eine Kur kann nur Erfolg haben, wenn sie möglichst lange fortgesetzt wird, mit ein paar Wochen ist hier nichts getan; das Wichtigste ist, daß Jod jahrelang, wenn allch mit kurzen, 8-14 tägigen Palısen, die allo sechs Wochen eingeschaltet werden, gegeben werdon kann. Dalauf hat schon vor längerer Zeit Erlenmeyer hillgewiesell. $D a ß$ bei einer so lange dallernden Medikation der Patient genau ïberwacht werden miB, liegt auf der Hand. Besonders beaclitet werden miissen Herz, Magen und Nieren.

Was zunächst das Her z betrifft, so zeigen viele Patienten, das ist lhnen ja aus der inneren Klilik bekannt, nach Jodkaliumdarreichung häufig oine ausgesplochene Besserung ihrer arteriosklerotischen Herzbescliwerden. Abel llach den Mitteilungen, welche uns kiirzlich $\mathrm{H}$ is gemacht hat, gibt es namentlich in Basel Fälle, wolche nach längerer Joddarreichıng init einer mohr oder minder starken Pulsbeschleunigung reagielen. Diese Pulsbeschleuniging habe ich unter auBerordentlich zahlreichen Fällen hier bisher nur einmal gesehen. Es ist möglich, daß es sich in den Hisschen Beobachtungen, wie er selbst auch annimmt, un eine regionäre Erscheinung laandelt. Alff jeden Fall miissen wir aber darauf achten.

Daß einzelne Patienten eine gewisse Idios ynkrasie gegen Jod haben lind sofort mit starkem Schnupion reagieren, ist Ihnen bekannt; ich habe diese Erscheinung nicht erwähnt, weil sie nicht so wichtig ist und viele Patienten trotzdem bei vorsichtigem Vorgehen lloch an das Jod gewöhnt werden können. Schwieriger liegen die Verlaältnisse, wenn der Magen versagt oder die Nierell Schwierigkeiten machen. Das heiBt für den elsten Fall, wenn der Appetit abnimint, für den letzteren Fall, wenı das Eiweib zunimmt. In solchen Fällen müssen wir sofort nit del- Jodnedikation anfhörenl. So selbstverständlich es ist, so inıß man bei der Veroldilung doch immer daraul allfmerksam merken, daß die Jodlösung nie pur, sondern inmer mit etwas Milch nlld am besten nach der Mahlzeit genommen wird.

Geht trotzdem die Nahrungsallfnahme deutlich zurïck, so müssen wir uns nach anderen Jodpräpalaten umsehen. Hier kommen in Betracht Sajodin lund Jodipin. Mit beiden Präparaton kann man dieselbe Wirkung wie mit Jodnatrinm oder Jodkalium erzielen. Aber man ist allch hierbei nie sicher, ob nicht der Magen allmählich versagt. Immer wieder kommen Patienten, welche auch hierbei über schlechten Geschmack und Nachlassen des Appetites klagen. Es scheinen hier individuelle Komponenten, welche wir noch nicht iibersehen können, eine Rolle zu spielen. Auf jeden Fall haben wir aber in diesen beiłen Präparaten eine schätzenswerte Hilfe, um in den Fällen, wo der Patient dringend nach einer Abhilfe verlangt, eine Aenderung in der Medikation ejntreten zu lassen. Im letzten halben Jahre habe ich allch alsgedehntele Velsuche mit einem Jodöl, Jothion, gemacht, das sich entschieden in den Fällen, wo man mit der innerlichen Darreichung kein Glück hat, zu bowähren scheint. Unangenehme Erscheinungen von seiten der Haut, wo es eingerieben wird, habe ich noch nicht gesehen.

In den Fällen, wo im Anschlub an einen Insult oder im AnschlnB an einen posthemiplegischen epileptoiden Zustand stärkere Verwirrıngs- und Desorientiertheitszustände mit einer gewissen Unruhe sich kombinieren, tut man gut, das Jod. präparat mit einem Brompräpara t zu kombinieren, am besten zu gleichen Teilen. Ist Beruhigung eingetreten, läßt man am besten den Bromanteil wieder weg. Nur in den Fällen, wo eine Komplikation mit ausgesprochener ateriosklerotischer Spätepilepsie vorliegt, wird man je nach Bedarf den Bromanteil längel bestehen lassen und eventuell anch steigern:

Die Art lnd Weise, wie das Jod wirkt, ist wissenschaftlich noch lange nicht klargelegt, so sicher allch die Empirie seine heilende Kraft bewiesen hat. Ich will Sie nicht mit langen theoretischen Erwägungen hierüber aufhalten, sondern nur hervorheben, daß dem Jod eine die Viskosität des Blutes herabsetzende Eigenschaft zukommt.

Mit Reeht werden Sie fragen, wie sind nun die Resultate nnserer Behandlung? Daß wir mit unserer Therapie überall da, wo Horde Substanz des Gehirns zerstört haben, eine Restitutio ad integrum nicht erzielen können, liegt auf der Hand, aber ebenso sicher ist, daß wir in den Fällen, wo wir die Behandinng gleich im Anfang der Krankheit beginnen können, Besserungen erzielen, die einer Heilıng gleichkommen, und auf jeden Fall dem Patienten ermöglichen, seine Berufsgeschäfte für längere und lange Zeit wieder aufzunehmen. Notwendig ist allerdings dabej, daß der Patient ständig unter ärztlicher Aufsicht steht und vor allen sclıädlichen Momenten, wie ich sie oben erwähnt habe, nach Möglichkeit bewahrt werden kann. Wir können entschieden bei einer nicht geringen Zahl von Patienten bei zeitigem Eingreifen das Leben unter leibliohem Wohlbefinden erheblich verlängeln. In den Fällen, wo bereits Insulte vorgelegen haben, können wir den Prozeb oft jahrelang aufhalten. Erfolgt auch keine völlige Zurückbildung der Lähmungserscheinıngen, so ist es doch nicht selten möglich, bei energischem Eingreifen den psychischen Verfall aufzuhalten. Selbstverständlich werden uns aber auch Fälle entgegentreten, bei denen unsere Therapie machtlos ist. Jch bin otwas genaller auf die Therapie eingegangen, weil ich die Hoffnung hege, daß Sie in all den Fällen, wo sich die nıehrfach erwähnte, ominöse Trias zeigt, nicht einfach abwarten, sondern therapeutisch eingreifen, um eventuell weiteres Unheil zu verhüten. 